\title{
An experimental comparison of cognitive versus traditional approaches to teaching the prepositions:
}

in, on, at 
I. Abstract

\section{Abstract}

When learning a new language, prepositions are as commonly used as they are difficult to master. In the Spanish learning context, the first and most common prepositions to be taught in Secondary Education are the units in, on, at. However, the fact that the teaching of these prepositions comes at an early stage does not mean that they are easy to learn. In fact, teaching materials have typically assumed that prepositions are used in a non-systematic way, thus dealing with them from a collocational perspective. Against that position, research in the field of Cognitive Linguistics (henceforth $\mathrm{CL}$ ) has demonstrated that only a small minority of prepositional uses are thoroughly idiomatic. According to this discipline, there exist two positions in relation to prepositional meaning, namely monosemy and polysemy. Following the latter perspective, Navarro i Ferrando (1998) has developed a theoretical model in which the meanings of the prepositions in, on and at can be described by radial networks containing spatial, force-dynamic and functional semantic elements.

In the present study, the effectiveness of this model in teaching the prepositions in, on, at has been tested. For this purpose, an experiment has been designed in which two groups of 4th-year ESO are taught the target prepositions following two different approaches: CL-based and traditional, respectively. Thus, it is hypothesised that, after the study, the group following the $\mathrm{CL}$ approach will present a higher improvement in their command of the target prepositions than the group taught by means of a traditional approach.

The overall results obtained in the experiment corroborate the hypothesis stated above, thus proving the advantages that a field such as CL can offer to the sphere of language teaching. However, the results also suggest the necessity of further research that helps to understand the extent to which factors such as motivation can influence the success of this approach.

Keywords: prepositions, polysemy, Cognitive Linguistics, radial network, proto-concept.

\section{Introduction}

When learning a new language, prepositions are as commonly used as they are difficult to master. In the Spanish learning context, the first and most common prepositions to be taught in Secondary Education are the lexical units in, on, at. However, the similarities between their meanings make them particularly difficult to distinguish for Spanish learners, who usually think that they can 
translate them into the "equivalent» in their mother tongue (Navarro i Ferrando 1998, 2000). In addition, teaching materials not only devote little space to dealing with prepositions, but also present them from a collocational approach. Because of this, learners are forced to learn them phrase by phrase, which implies a great amount of rote learning to get familiar with only a small set of meanings (Lindstromberg 1996, 1998). However, in the last decades it has been demonstrated that only a small minority of prepositional uses are thoroughly idiomatic. A field which has paid much attention to this topic is that of Cognitive Linguistics, and more specifically Cognitive Semantics (henceforth CS), both from a theoretical and a pedagogical perspective (Boers 2013; Boers and Demecheleer 1998; Bratož 2014; Feist 2000; Johnson 1987; Lakoff 1987; Lindstromberg 1996, 1998; Navarro i Ferrando 1998, 2000, 2006; Campoy and Caballero 2001; Song 2013; Tyler and Evans 2003).

In the light of that research, the aim of this paper is to test the effectiveness in teaching the prepositions in, on, at of an approach based on a theoretical model developed by Navarro i Ferrando (1998), following a CL approach to prepositional semantics. For this purpose, an experiment has been designed in which two groups of $4^{\text {th }}$-year ESO students are taught the prepositions in, on and at from two different perspectives: one, following the $\mathrm{CL}$ approach mentioned above; the other, by means of a traditional approach based on collocations. Taking into account the characteristics of the two groups, our initial hypothesis is that, after the study, the group following a $\mathrm{CL}$ approach will present a higher improvement in their command of the target prepositions than the group taught by means of a traditional approach. If this hypothesis is met, the present study could provide further evidence of the benefits that the field of $\mathrm{CL}$, and particularly the cognitive approach to prepositional semantics, may offer to the world of language pedagogy.

\section{Theoretical framework}

In English, prepositions are the main tool to describe spatial relations. From a $\mathrm{CL}$ point of view, language is considered to reflect the real world, so that spatial relations expressed linguistically are conceptualisations or abstractions of physical relations. The perception of recurrent experiences conjugating the same aspects of space allows for humans to conceptualise spatial scenes in the form of a series of image schematic structures (Johnson 1987; Lakoff 1987; Navarro i Ferrando 2012). Image schemas are dynamic structures based on bodily experience that appear first in children's prelinguistic experience and are successively applied in order to make sense of new experiences. In terms of their image schematic configuration, spatial concepts are relational and need two other 
entities in the construal event for conceptualisation to take place

(Langacker 1987; Navarro i Ferrando 1998, 2000, 2006; Silvestre López and Navarro i Ferrando 2007). These are the trajector (henceforth $\mathrm{Tr}$ ) and the landmark (henceforth $\mathrm{Lm}$ ), whose relationship is asymmetrical. The former is the localised entity; although its name suggests movement, it appears both in static and dynamic relations. The latter, being the complement of the preposition, functions as background or reference point for the Tr.

\subsection{Traditional approaches to prepositions}

Navarro i Ferrando (1998, 2000, 2006) classifies the main descriptions of prepositional meaning into two different positions. On the one hand, a position based on monosemy, which defines prepositional meaning as a core sense and presents two trends. In one trend, the core sense determines all the uses of a preposition, whereas the context provides meaning aspects extrinsic to the spatial concept. In the other trend, the core sense is present in all the contexts where a preposition occurs. These introduce meaning nuances that can be ascribed to the preposition. On the other hand, a second position considers prepositions as polysemous items, with both prototypical and non-prototypical senses. These can be derived from a basic image-schema by means of family resemblances and image schema transformations.

In the core sense approaches, the description of in, on, at has followed topological or geometric configurations. However, descriptions based on geometry have a series of limitations. On the one hand, there exists a series of spatial usages which cannot be explained by this type of accounts. On the other hand, they are unable to explain the reasons why it is possible to describe particular scenes using different prepositions without a change in the spatial relation between the $\mathrm{Tr}$ and the $\mathrm{Lm}$. To this, Navarro i Ferrando adds that prepositions like in, on and at can be used with any type of $\mathrm{Lm}$ dimensionality $(1998,2000,2006)$.

\subsection{The polysemy approach to prepositions}

Given the inadequacy of topological approaches in determining the type of relationship established between two entities, the polysemy approach tries to introduce further aspects to the analysis of relational concepts. In relation to topology, the visual perception of objects gives the speaker clues for establishing and conceptualising topological relations such as coincidence, contact, inclusion, proximity, etc. Regarding force-dynamics, the human experience of self-motion and object motion provides the clues for conceptualising patterns of interaction in terms of force-dynamics. As concerns function, the experience of the effects of interaction, as 
well as the consequences of those effects, give rise to functional patterns in the conceptualisation of spatial relationships between different entities (Navarro i Ferrando 2006, 171). The consideration of these dimensions in the conceptualisation of spatial relations would be in line with one of the main principles of $\mathrm{CL}$, i.e. the embodiment hypothesis (Lakoff 1987; Varela et al. 1981). This perspective claims that human conceptualisation of reality is both constrained by our perceptual and cognitive capacities, and strongly rooted in bodily experience as well as in physical and social interaction. In other words, "the conceptualisation of both forcedynamic and functional relationships is as primary as the conceptualisation of topological ones in the acquisition of spatial concepts» (Navarro i Ferrando and Tricker 2001, 296).

Following the polysemy approach, Navarro i Ferrando (1998, $2000,2006)$ describes the primary meanings of the prepositions in, on, at as conceptual schemas or proto-concepts conformed by the three dimensions detailed above. "Proto-concepts are familyresemblance configurations where some aspects may be focused upon, while others constitute a background in the conceptualisation of a particular situation» $(2006,173)$. From these proto-concepts, specialised and extended meanings are derived, resulting in a semantic structure shaped as a radial network. In this network, the proto-concept is situated at the centre, and the peripheral meanings arise by its extension through the topological, dynamic and functional conceptual regions conjugated in the spatial relationship. The mechanisms that produce these derived meanings (i.e., polysemy) are, according to Navarro i Ferrando $(1998,2006)$ shifts, implying slight modifications of the conceptual schema (e.g. rotation), partial sanction, giving salience to particular configurations (i.e. specialisation of meaning), and metaphorical mappings from the physical to the abstract domains, prompting figurative meanings.

\subsection{Theoretical background}

In $\mathrm{CL}$, the experience gained through the cognitive and perceptual capacities involved in human interaction with the world determines the way in which language works. Taking this into account, some linguistic phenomena are more likely to occur than others because of their coherence with human experience, i.e., they are "motivated» (Boers 2013, 211). However, this motivation is not always transparent for language learners. According to the polysemy approach, prepositions are an example of motivated language items, in which the different senses of each spatial particle derive from a primary sense and extend conforming a semantic network. However, English teaching materials, and teachers in general, have tended to treat the semantics of prepositions from a "collocational approach" (Lindstromberg 1996, 227). This means that learners have not been 
shown the links between the different meanings of prepositions, but rather have had to memorise them one by one, a practice that implies lots of rote learning. Against that position, the advantage of the polysemy approach to prepositions in terms of pedagogy is that it can help learners to deal with their semantic structure as an overall meaning conformed by a relatively small number of related meanings more or less systematically combined. Thus, it allows for enquiries into what individual words mean, as opposed to what phrases they occur in, what can lead to a decrease in the memorisation effort that students have to perform (Lindstromberg 1996).

\subsection{A model for the polysemy of in, on, at}

To finish this section, an outline of Navarro i Ferrando's (1998, 2006) models for the multimodal semantic structure of the prepositions in, on, at is presented below.

The conceptual schema instantiated by in can be defined by the term CONTAINMENT. Human experience with this type of schema determines that it is composed of the following elements. According to the force-dynamic configuration, the Tr may be either static within the interior region defined by the Lm, or dynamic, moving within the interior or from the exterior to the interior of the $\mathrm{Lm}$. In any case, the Lm prevents the Tr from moving outside. Regarding the topological configuration, the Lm defines an interior space where the Tr, being smaller, is located. The functional dimension defines the relationship of control of the Lm over the $\mathrm{Tr}$ as one of reclusion or protection.

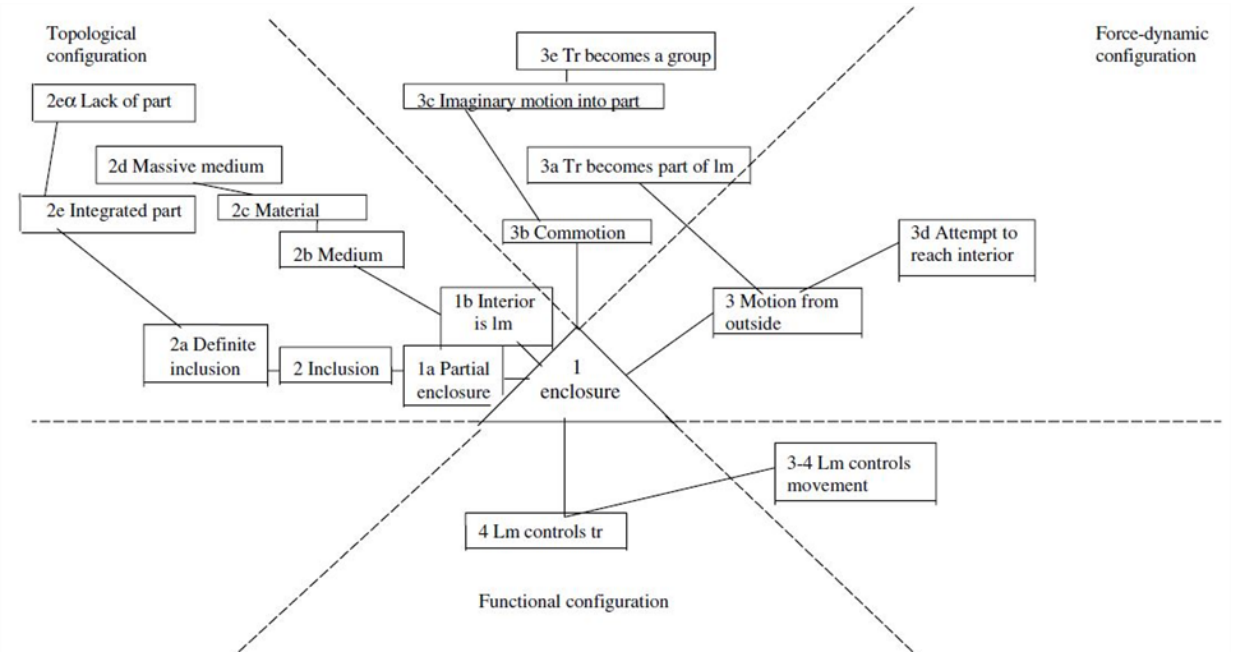

Figure 1. Radial category for in (Navarro i Ferrando 1998, 266)

As mentioned above, the semantic structure of prepositions can be represented as a conceptual schema with the shape of a radial network (Figure 1). In the radial network for in, the central element corresponds to the proto-concept of ENCLOSURE, renamed CONTAINMENT (Navarro i Ferrando 2006). This is also the primigenial 
sense, as it is considered to be the first usage learnt by children. The senses surrounding the central meaning, i.e. peripheral senses, are assumed to have been acquired later, and emerge through meaning extensions from the central meaning. These extensions are first generated through image-schema transformations, and then by means of mechanisms such as "blending spaces, semantic bleaching, or double highlighting» for further specialisations $(1998,145)$. Each prepositional usage gives salience to particular perceptual aspects, which give rise to the conceptual regions where the different senses extend, namely topological, force-dynamic and functional. These regions do not present clear-cut borders, but rather form a continuum where the distance between the different senses reflects the extent to which they are related.

The conceptual schema of on can be defined by the term SUPPORT. The configuration defined by human experience with this schema can be described as including the following elements. On the one hand, the force-dynamic dimension determines that the resting side of the $\mathrm{Tr}$ and its orientation towards the $\mathrm{Lm}$ define the motion axis. Prototypically, this corresponds to the vertical axis with respect to the human canonical standing position, so the Tr exerts force downwards; however, the vertical axis may rotate in situations where the position of Tr's resting side does not coincide with the human one. According to topological configuration, the relationship between the $\mathrm{Tr}$ and the $\mathrm{Lm}$ is one of contact, involving the outside part of the Lm and the resting part of the Tr. Last, in relation to the functional dimension, the Tr holds control of the situation. It may be prototypically self-control, or motion control, which may be extended to the $\mathrm{Lm}$.

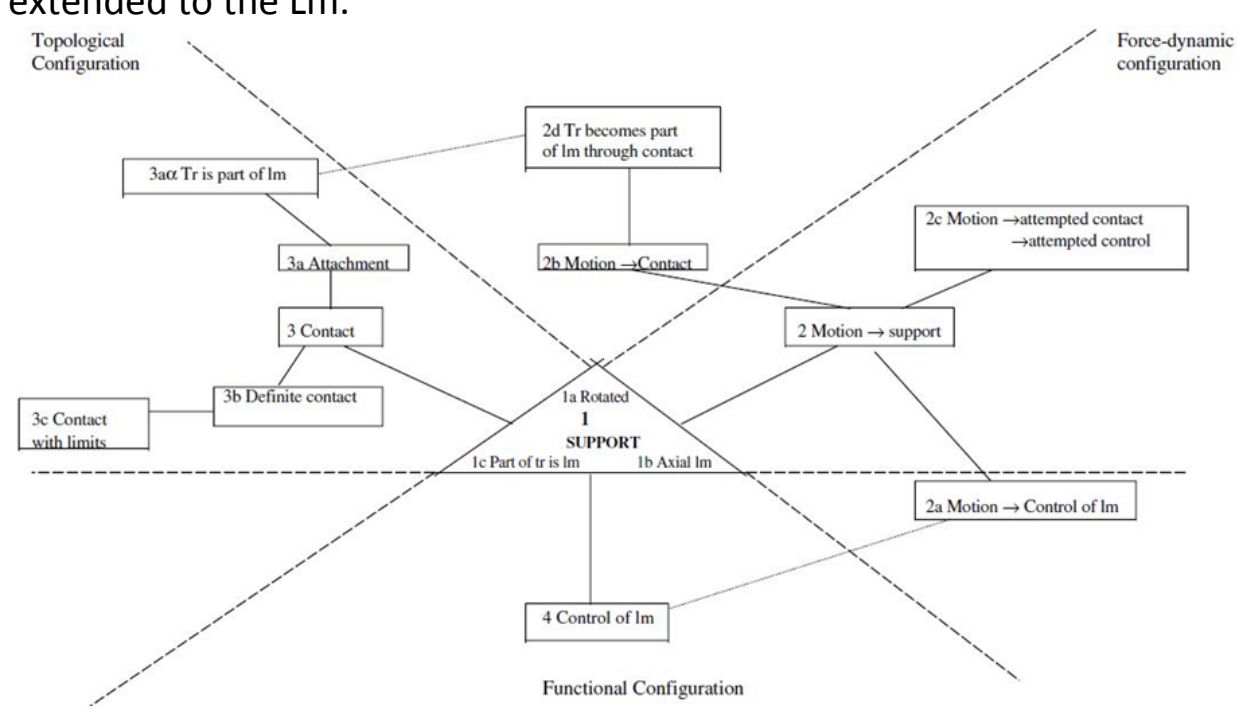

Figure 2. Radial category for on (Navarro i Ferrando 1998, 219)

In the radial network representing the semantic structure of on (Figure 2), the proto-concept of SUPPORT, which is also considered to be the first usage acquired by children, is situated at the centre of 
the net. The peripheral senses are situated around the central meaning. This situation indicates both that native speakers learn them later, and that they emerge from the central meaning through meaning extensions. At the first level of specialisation, these extensions are first generated through image-schema transformations highlighting one of the perceptual aspects (topological, force-dynamic, or functional). Further specialisations take place by means of mechanisms such as «blending spaces, semantic bleaching, or double highlighting» (1998, 145). Thus, peripheral meanings expand across the conceptual regions emerging from the highlighted perceptual aspects. The different conceptual regions are not fully separated, as the boundaries between them are merged. The relation between senses belonging to different conceptual regions is measured in terms of the distance separating them.

The conceptual schema for at can be defined by the term ENCOUNTER. Based on human bodily experience with this schema, at conjures the following elements. According to the force-dynamic configuration, the motion axis is defined by the functional front of the $\operatorname{Tr}$ and its orientation towards the Lm. The human canonical standing position determines that this axis is prototypically horizontal. As regards the topological configuration, the relationship between the $\mathrm{Tr}$ and the Lm is of contiguity, which does not necessarily imply contact. Finally, in relation to the functional dimension, there is certain intentionality of the $\operatorname{Tr}$ with respect to the $\mathrm{Lm}$ in order to use, manipulate, or affect it.

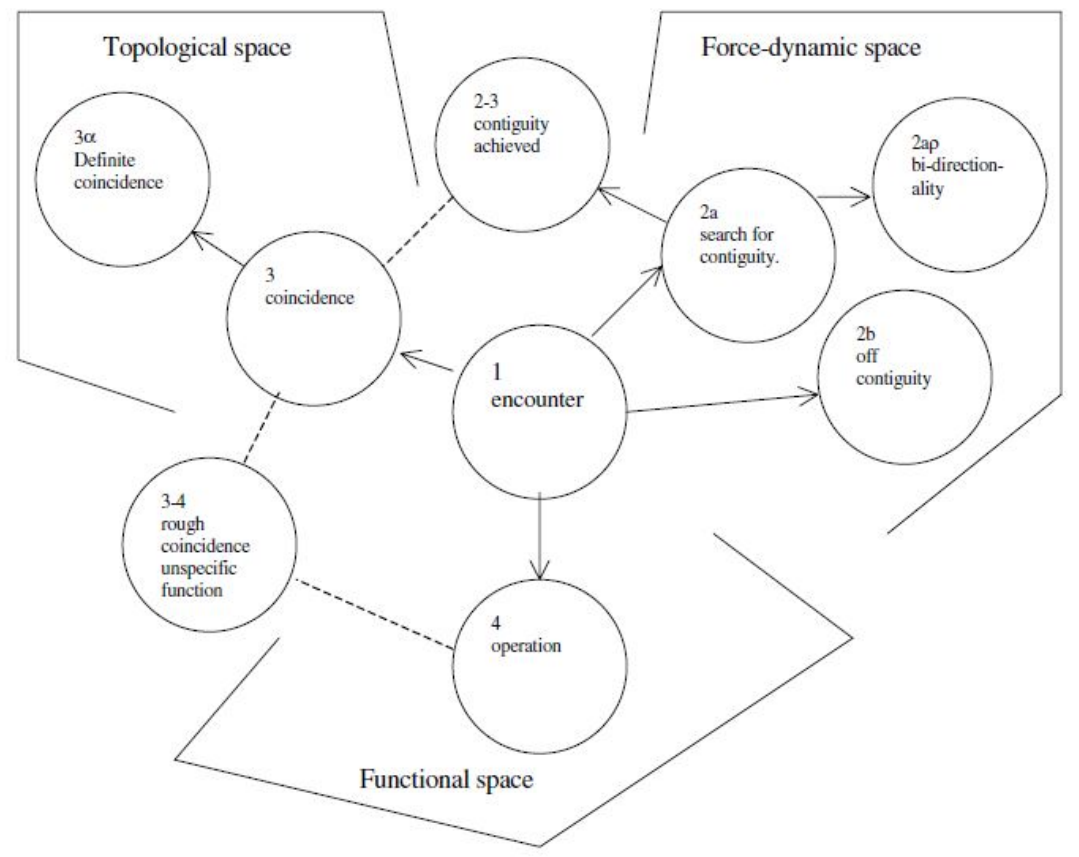

Figure 3. Radial category for at (Navarro i Ferrando 1998, 174) 
The polysemic structure of the preposition at is represented in the radial category corresponding to Figure 3 . The primigenial sense, which is the first meaning that children learn, is situated at the centre of the network. Around the central sense, the peripheral meanings, acquired in later stages, extend through the topological, force-dynamic and functional regions. The peripheral senses corresponding to the first level of specialisation are generated by highlighting one of the perceptual aspects that give rise to the conceptual regions. Other mechanisms, such as blending spaces, semantic bleaching and double highlighting give rise to further specialisations. The conceptual regions across which the different specialised meanings do not present clear-cut borders, so the extension from one sense to another is gradual. Accordingly, the extent to which senses situated in different regions are related to each other is represented by the distance that separates them.

\section{Methodology}

The present study primarily attempts to examine whether the $\mathrm{CL}$ method based on a theoretical model developed by Navarro i Ferrando (1998) is more effective in teaching the prepositions in, on, at than a traditional approach based on collocations. For this purpose, the hypothesis that a group of students taught by means of a $\mathrm{CL}$ methodology will show greater improvement in their command of the target prepositions than another group taught following a collocational approach has been tested by means of an experiment. In the present section, an account of the research process followed in this study is provided.

\subsection{Participants}

During my Practicum, I have taught English to two 4ESO classrooms. The average student profile in both classrooms is that of a 16 to 18 year old individual who can speak Spanish and Catalan, apart from English and, in some cases, other native languages. However, a number of differences can be observed between the two classroom contexts. Regarding 4ESO KB, it is a group of 27 students from two different groups, $\mathrm{B}$ and $\mathrm{K}$. Their overall language command is higher than the one in $4 \mathrm{ESO} \mathrm{ACL}$. In fact, most of the students in this classroom were part of high-performance English groups in previous academic years. In terms of motivation, the classroom shows a positive attitude towards learning, and most of them are planning on continuing their studies in the following years. In relation to $4 \mathrm{ESO} \mathrm{ACL}$, it is composed of 16 students, most of which were part of low-performance English groups, and their overall language command is lower than the one in group 4ESO KB. In general, their motivation towards learning, and particularly towards learning 
English, is also lower than in the other group, as they are expected to end their studies or take vocational training as soon as they can, so they feel that they will not need English in the future. In order to compensate for the differences in level and motivation, the experimental methodology was used in the less advantaged group, 4ESO ACL, whereas $4 \mathrm{ESO}$ BK became the control group.

\subsection{Materials}

Two types of materials were used. On the one hand, the teaching materials used as support for the theoretical classes; on the other hand, the pre-tests and post-tests, or questionnaires, used at the beginning and at the end of the research process.

Regarding the questionnaires, two models were created for this experiment, namely $\mathrm{A}$ and $\mathrm{B}$. Both models were used as pre-test and post-test. Each of these handouts contained the following tasks. Task 2 was a fill-in-the-gap activity that required the students to choose the most correct preposition to complete a set of 30 sentences, all extracted from Units 121 to 125 in Murphy's English Grammar in Use $(2012,242-252)$. This was made to ensure the appropriateness of the exercise to the students' English level, which at the 4ESO stage is assumed to be intermediate. Task 3 was also a fill-in-the-gap activity, but on this occasion the sentences lacked both the preposition and its complement. The complements to be used in the different gaps were scrambled on a table, and students had to select the most suitable one for each sentence. The six sentences used in this exercise were also extracted from Murphy's (2012) units devoted to prepositions.

As regards the theoretical materials, these consisted of a series of Powerpoint presentations and practical exercises. These exercises were taken from Song (2013), where the author also compares a CL with a traditional approach to teaching the prepositions in, on, at. With respect to the control group, 4ESO BK, the target prepositions were taught from a collocational perspective. For this, and following Song's (2013) example of a traditional approach, the different collocational contexts in which the prepositions can be used were arranged in terms of a definition elicited by the particular utterances shown in the Powerpoint. Concerning the experimental group, 4ESO $A C L$, students were introduced to $C L$ and the main concepts they needed to understand this approach. After this, they were presented a simplified version of the proto-concepts developed by Navarro i Ferrando (1998) to explain the meanings of in, on, at.

\subsection{Procedure}

For the experiment, students were asked to fill in a questionnaire as a pre-test. Two versions of the questionnaire, 
namely $A$ and $B$, were distributed around the classrooms so that no students sitting contiguously had the same model, in order to avoid cheating. After the pre-test and before the post-test, two sessions were devoted to teaching the prepositions in, on, at in both 4ESO classrooms. In these, two different methodologies were used: a $\mathrm{CL}$ approach in the experimental group (4ESO ACL), and a traditional approach in the control group (4ESO BK). In order to ensure the same learning opportunities, both methodologies were applied under similar conditions. In the post-test session, the handouts were the same as in the pre-test, but students who had previously taken model $A$, now received model $B$, and vice-versa.

In order to test the main hypothesis in this study, a comparison was made between the achievements obtained by students in both groups in the initial and final questionnaires. However, as some members from the two groups had missed either the pre-test or the post-test, the number of valid samples was lower than the number of people in each classroom. Thus, in 4ESO BK -the control group- the final amount was 21 , while in $4 \mathrm{ESO} A C L$-the experimental grouponly 11 samples were valid.

\section{Results}

The results of the experiment are presented in the tables below. These figures have been created in order to compare two dimensions of the data collected in the study. One dimension is the number and quality of learners' answers in the pre-test and post-test; the other, the students' overall performance in the initial and final questionnaires.

On the one hand, the percentages of correct $(\checkmark)$, incorrect $(X)$ and blank $(\varnothing)$ answers provided to Task 2, Task 3, and to the combination of both tasks (Task 2 + Task 3), are displayed in Table 1 (control group) and Table 2 (experimental group). In these, the first row shows the answers corresponding to the pre-test, while the ones relating to the post-test are situated on the second row. At the bottom of the tables, the difference between the percentages corresponding to the pre-test and the post-test (Variation) is illustrated. A negative Variation indicates that the number of items corresponding to an answer type (for instance, correct answers) in the post-test is lower than the number of the same answer type in the pre-test, and vice-versa.

Table 1. Percentages of correct, incorrect and blank answers to Task 2, Task 3, and

Task $2+$ Task 3 in the pre-test and the post-test, and their variation between the two tests (control group)

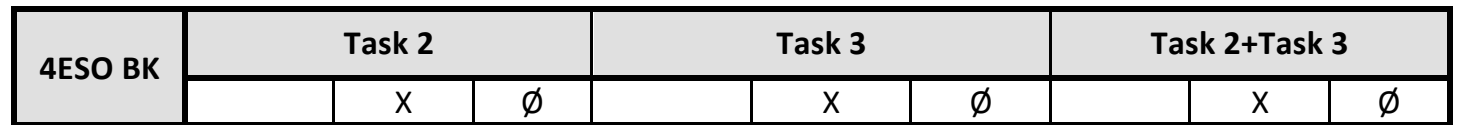




\begin{tabular}{|c|c|c|c|c|c|c|c|c|c|}
\hline Pre-test & $47.9 \%$ & $50 \%$ & $2.1 \%$ & $36.25 \%$ & $60 \%$ & $3.75 \%$ & $45.3 \%$ & $52.2 \%$ & $2.5 \%$ \\
\hline Post-test & $47.5 \%$ & $52.5 \%$ & $0 \%$ & $36.25 \%$ & $63.75 \%$ & $0 \%$ & $45 \%$ & $55 \%$ & $0 \%$ \\
\hline
\end{tabular}

Table 2. Percentages of correct, incorrect and blank answers to Task 2, Task 3, and Task $2+$ Task 3 in the pre-test and the post-test, and their variation between the two tests (experimental group)

\begin{tabular}{|c|c|c|c|c|c|c|c|c|c|}
\hline \multirow{2}{*}{ 4ESO ACL } & \multicolumn{3}{|c|}{ Task 2 } & \multicolumn{3}{c|}{ Task 3 } & \multicolumn{3}{c|}{ Task 2+Task 3 } \\
\cline { 2 - 10 } & & $\mathrm{X}$ & $\varnothing$ & & $\mathrm{X}$ & $\varnothing$ & & $\mathrm{X}$ & $\varnothing$ \\
\hline Pre-test & $42.2 \%$ & $56.5 \%$ & $1.3 \%$ & $27.3 \%$ & $52.3 \%$ & $20.4 \%$ & $38.9 \%$ & $55.6 \%$ & $5.5 \%$ \\
\hline Post-test & $40.9 \%$ & $58.4 \%$ & $0.7 \%$ & $36.4 \%$ & $54.5 \%$ & $9.1 \%$ & $39.9 \%$ & $57.5 \%$ & $2.6 \%$ \\
\hline
\end{tabular}

On the other hand, a comparison has also been made in relation to the number of students in each classroom who have achieved the same, better or worse results in the pre-test and the post-test. These are illustrated in Table 3 (control group) and Table 4 (experimental group). These tables represent the difference between students' performance in Task 2, Task 3, and to the combination of both tasks (Task $2+$ Task 3 ) in the pre-test and the post-test. In this case, the proportion of students who have achieved the same, worse or better results is represented as a number and as a percentage.

Table 3. Number and percentage of students who have improved, worsened or obtained equal results in Task 2, Task 3, and Task 2 + Task 3 together between the pre-test and the post-test (control group)

\begin{tabular}{|c|c|c|c|c|c|c|c|c|c|}
\hline \multirow{2}{*}{ 4ESO BK } & \multicolumn{3}{|c|}{ Task 2 } & \multicolumn{3}{c|}{ Task 3 } & \multicolumn{3}{c|}{ Task 2+Task 3 } \\
\cline { 2 - 12 } & better & worse & equal & better & worse & equal & better & worse & equal \\
\hline Number of students & 9 & 7 & 4 & 6 & 7 & 7 & 7 & 8 & 5 \\
\hline Percentage & $20 \%$ & $35 \%$ & $45 \%$ & $30 \%$ & $35 \%$ & $35 \%$ & $35 \%$ & $40 \%$ & $25 \%$ \\
\hline
\end{tabular}

Table 4. Number and percentage of students who have improved, worsened or obtained equal results in Task 2, Task 3, and Task 2 + Task 3 together between the pre-test and the post-test (experimental group)

\begin{tabular}{|c|c|c|c|c|c|c|c|c|c|}
\hline \multirow{2}{*}{ 4ESO ACL } & \multicolumn{3}{|c|}{ Task 2 } & \multicolumn{3}{c|}{ Task 3 } & \multicolumn{3}{c|}{ Task 2+Task 3 } \\
\cline { 2 - 11 } & better & worse & equal & better & worse & equal & better & worse & equal \\
\hline $\begin{array}{c}\text { Number of } \\
\text { students }\end{array}$ & 5 & 5 & 1 & 5 & 3 & 3 & 4 & 6 & 1 \\
\hline Percentage & $45.45 \%$ & $45.45 \%$ & $9.1 \%$ & $45.45 \%$ & $27.27 \%$ & $27.27 \%$ & $36.4 \%$ & $54.54 \%$ & $9.1 \%$ \\
\hline
\end{tabular}

\section{Discussion}

On the one hand, in relation to the variation between the number of correct, incorrect and blank answers emitted in the two classes in the pre-test and the post-test, the results obtained from 
Task 2 show that the proportion of correct answers provided by students in the control group both in the pre-test $(47.9 \%)$ and the post-test $(47.5 \%)$ is higher than the ones corresponding to the experimental group ( $42.2 \%$ and $40.9 \%$, respectively). In Task 3, the control group have achieved an equal percentage of correct answers in the initial and final questionnaires. However, their proportion of incorrect answers has increased a $3.75 \%$. In contrast, the experimental group, who obtained a lower percentage in the pretest, have achieved slightly better results than 4ESO BK (control group) in the post-test. Thus, even if $4 \mathrm{ESO}$ ACL (experimental group) have experienced a $2.2 \%$ increase in their number of incorrect answers, the variation in their proportion of correct answers (+9.1\%) is much higher than that. Considering the results obtained from Task $2+$ Task 3 together, these do not seem very positive, as both groups have reached an overall higher proportion of wrong answers than of correct ones. Despite that, the outcome of the experiment is more positive in the experimental group, in that their number of correct answers has seen a greater increase than in 4ESO BK (control group). In consonance with that, the rise in the number of incorrect answers has been smaller in the experimental group as well.

In relation to our initial hypothesis, the results from Task 2 seem to contradict it, whereas the results from Task 3 and Task 2+Task 3 considered together comply with it. These data seem to contradict each other; however, there is a factor that may explain the experimental group's unsuccessful performance in Task 2: motivation. In the description of the participants, it has been pointed out that students in the experimental group are characterised by an overall lack of motivation. During the research process, students in the control group have not been openly enthusiastic neither about the questionnaires nor the theoretical explanations. However, their behaviour and attitude towards learning could be described as a fairly positive. In contrast, the overall attitude in the experimental group has been negative, as several students in 4ESO ACL showed indifference, or even open rejection, towards the questionnaires and the theoretical classes. This contrast between the two groups can be seen, for example, in the fact that a much higher percentage of the experimental group has left questions unanswered (i.e. blank) in the questionnaires, even in the post-test. Extrapolating these attitudes to the scope of the activities, Task 2 is much longer than Task 3. Therefore, students may have found that completing the former is more boring than completing the latter. In addition, Task 2 constitutes a classic example of fill-in-the-gap activity in which the sentences are mechanically answered with one preposition. In contrast, Task 3 involves the necessity of combining the prepositions with another element. This fact might have made the students consider the completion of Task 3 as more appealing than the 
mechanical selection of a preposition in Task 2. In other words, it could be argued that the students' negative attitude towards the questionnaires, and particularly towards Task 2, has had a negative influence on the experiment's results, most notably in the experimental group. In this sense, it could be assumed that participants in the experimental group have paid less attention, and therefore have provided a greater number of wrong answers to the task that engaged their motivation the least.

On the other hand, the outcomes of the experiment can also be assessed in terms of the percentage of students from the two groups who have improved, worsened, or obtained equal results in the pretest and the post-test. Concerning Task 2, the proportion of students who have improved in the experimental group (45.45\%) is slightly higher than in the control group (45\%). However, an equal fraction of students have obtained negative results in the experimental group (45.45\%), while in the control group the difference between the students who have improved and worsened is more obvious (45\% and 35\%, respectively). Regarding student performance in Task 3 in the two groups, the class in which more people have improved is the experimental group. In fact, not only the proportion of successful students in this group is greater than of those who have not succeeded, but also than their counterparts in the control group. In addition, in the control group there is a greater fraction of students who have worsened (35\%) than of students who have improved (30\%). Finally, the data gathered from Task 2 + Task 3 together indicate that the proportion of students who have improved in the experimental group (36.4\%) is higher than in the control group (35\%). However, the number of worse performances in the experimental group is also greater nonetheless (54.5\%), as in the control group there is a less sharp proportion of students who have done worse between the pre-test and the post-test (40\%).

Summarising, the data gathered in relation to the number of students who have achieved better, worse or equal results between the pre-test and the post-test in each group agree with our initial hypothesis, that is, that the group following a CL approach would improve more their command of the prepositions in, on, at than the group following a traditional approach. Indeed, in Task 2, Task 3 and Task $2+$ Task 3 , the percentage of students who have improved their results between the pre-test and the post-test is higher in the experimental group. These results would also be in line with the ones obtained by Song (2013) in his experimental comparison between a $\mathrm{CL}$ and a traditional approach to teaching the prepositions in, on, at.

\subsection{Conditions of the experiment: drawbacks}

Despite the fact that the results obtained in the study agree with our initial hypothesis, it must be also pointed out that both the 
experimental and the control group present an important fraction of students who have not achieved better results in the pre-test than in the post-test, and that the percentages of students who have worsened are greater in the experimental group. In relation to this situation, a series of factors may have had an influence on the students' performance between the initial and final questionnaires. These are motivation, confidence, class attendance, timing, and the teaching materials.

As regards motivation, its effects, which have been most notable in the experimental group's performance, it has been suggested that the experimental group's lack of motivation has lead the students in this class to demonstrate a negative attitude towards learning, which has been translated into a worse performance when completing an activity the nature of which might not engage their attention (Task 2). Another way in which motivation might have affected students' performance is related to its connection with their confidence. It could be argued that the existence of a more positive attitude towards the experiment in the control group than in the experimental group may be explained in terms of students' confidence. Since most of the students in the control group have a good English level, they feel confident about it. This confidence might, in turn, generate motivation towards learning, seen as a pleasant experience. However, the situation in the experimental group is the opposite, as they think of themselves as having a poor command on the English language, which is translated into a more negative attitude towards learning.

Class attendance is another factor that might have had a particularly negative influence on the experimental group's results. Indeed, several students missed part of the theoretical explanations. This fact can be considered to have affected negatively both, these students' results, as well as the outcomes of the whole class. On the one hand, the students who missed the lessons can be expected to have achieved a poorer understanding of the theoretical approach they were explained, and thus on the use of the target prepositions. On the other hand, the lack of understanding can also have affected the students' confidence, and therefore have derived into worse results affecting both, their individual performance as well as their performance at the class level.

The lack of sufficient time to review the theory dealt with in the two groups might have influenced the students' performance negatively as well. Indeed, there were time constraints in relation to the number of sessions available for the experiment. In addition, the experiment coincided with a period in which students need to devote most of their time preparing exams for the rest of subjects, thus leaving other matters unattended. 
Finally, the way in which the materials have been presented to students can also be considered to have affected the results of the experiment. In the experimental group, the theoretical explanations related to the $\mathrm{CL}$ approach to the semantics of the prepositions in, on, at have been presented by means of a language that can be considered as too specialised for $4 \mathrm{ESO}$ students. In fact, it could be said that students have been taught a $\mathrm{CL}$ theory of prepositional meaning, rather than having learnt from exercises adapting said $\mathrm{CL}$ theory to their instructional level. The lack of a proper adaptation of the theoretical materials to the students is due both to the time constraints mentioned above in relation to the period available to implement the experiment, and to the lack of materials to be used as reference.

\section{Conclusion}

The results obtained in this study corroborate our initial hypothesis in terms of two dimensions. On the one hand, the variation between the number of correct, incorrect and blank answers emitted in Task 3 and Task 2 + Task 3 in the pre-test and the post-test by students in the experimental group has been more positive than in the control group. In addition, the class in which a greater number of students have improved their achievements between the initial and the final questionnaires has also been the experimental group. More importantly, these results demonstrate that the field of $\mathrm{CL}$ has yet much to offer to the world of language pedagogy, and particularly to the teaching of prepositions.

However, the data gathered in relation to the number of students who have improved their results in Task 2 have been more positive in the control group. In the light of these results, it has been argued that the difference in motivation between the students in the experimental and the control group has played an important role. Similarly, a great proportion of students in the control and experimental groups have obtained worse results in the post-test than in the pre-test. Thus, this situation has been argued to have been provoked by the coincidence of a series of factors in the experimental conditions that might have had a negative impact on the results. These factors are motivation, confidence, class attendance, timing, and the design of the teaching materials.

The main conclusion that can be extracted from these apparently contradictory results is that furhter research is needed in order to corroborate whether, and to what extent, the negative results obtained in the experiment can be attributable to the factors mentioned above. Studies similar to this one, and others carried out longitudinally and with a larger number of participants need to be conducted. The difficulty to find and design suitable materials for the 
implementation of the theoretical principles of $\mathrm{CL}$ in Secondary Education suggests the necessity of research projects that follow the lines opened by Boers (2013), Johansson Falck (2018), Lindstromberg (1996, 1998), Navarro i Ferrando, Campoy and Caballero (2001) or Song (2013).

\section{References}

Boers, Frank. 1996. Spatial prepositions and metaphor: a cognitive semantic journey along the up-down and the front-back dimensions. Tübingen: Gunter Narr Verlag.

Boers, Frank and Murielle Demecheleer. 1998. "A cognitive semantic approach to teaching prepositions». ELT journal 52 (3): 197-204.

Boers, Frank. 2013. "Cognitive Linguistic approaches to teaching vocabulary: Assessment and integration». Language Teaching 46 (2): 208-224.

Bratož, Silva. 2014. "Teaching English locative prepositions: a cognitive perspective». Linguistica 54 (1): 325-337.

Feist, Michelle. 2000. "On in and on: An investigation into the linguistic encoding of spatial scenes». PhD dissertation, Northwestern University.

Hornby, Albert et al. 2005. Oxford Advanced Learner's Dictionary. Oxford: Oxford University Press.

Johansson Falck, Marlene. 2018. «Embodied experience and the teaching and learning of $L 2$ prepositions: A case study of abstract in and on". In What is Applied Cognitive Linguistics? Answers From Current SLA Research, edited by Andrea Tyler, Lihong Huang and Hana Jan, 285-302. Berlin and Boston: De Gruyter Mouton.

Johnson, Mark. 2013. The body in the mind: The bodily basis of meaning, imagination and reason. Chicago: University of Chicago Press.

Lakoff, George. 1987. Women, Fire, and Dangerous Things: What Categories Reveal About the Mind. Chicago: University of Chicago Press.

Langacker, Ronald W. 1987. Foundations of Cognitive Grammar. Volume I: Theoretical Prerequisites, Stanford: Stanford University Press.

Lindstromberg, Seth. 1996. "Prepositions: meaning and method». ELT Journal 50 (3): 225-236.

Lindstromberg, Seth. 1998. English prepositions explained. Amsterdam and New York: John Benjamins Publishing. 
Murphy, Raymond. 2012. English Grammar in Use. A self-study reference and practice book for intermediate learners of English. Cambridge, UK: Cambridge University Press.

Navarro i Ferrando, Ignasi. 1998. A Cognitive Semantics Analysis of the Lexical Units $A T, O N$, and IN in English. PhD dissertation, Universitat Jaume I.

Navarro i Ferrando, Ignasi and María Carmen Campoy. 2001. "Thinking with English prepositions and adverbs». In Docència universitària: avanços recents: Primera Jornada de millora educativa de la Universitat Jaume I, edited by Universitat Jaume I, 243-252. Castelló de la Plana: Publicacions de la Universitat Jaume I.

Navarro i Ferrando, Ignasi and Deborah Tricker. 2001. "A Comparison of the use of at, in, and on by EFL students and native speakers». Revista Española de Lingüística Aplicada 14: 295-324.

Navarro i Ferrando, Ignasi. 2006. "On the meaning of three English prepositions». In In-roads of Language: Essays in English Studies, edited by Ignasi Navarro i Ferrando and Nieves Crespo. Castelló de la Plana: Publicacions de la Universitat Jaume I.

Navarro i Ferrando, Ignasi. 2012. «Exploring the Lexical Representation of English Particles in the Lexical-Constructional Model». In Cognitive Linguistics between Universality and Variation, edited by Mario Brdar, Ida Raffaelli and Milena Žic Fuchs, 137-156. Newcastle upon Tyne: Cambridge Scholars.

Silvestre López, Antonio José and Ignasi Navarro i Ferrando. 2007. «Diachronic Change in the Semantic Configuration Of Six VerbPreposition Combinations». Studia Universitatis Petru Maior Serie Philologia, 6: 156-171.

Song, Xin. 2013. A cognitive linguistic approach to teaching English prepositions. PhD dissertation, University of Koblenz-Landau.

Tyler, Andrea and Vivan Evans. 2003. The Semantics of English Prepositions: Spatial scenes, embodied meaning, and cognition. Cambridge: Cambridge University Press.

Varela, Francisco, Eleanor Rosch, and Evan Thompson. The embodied mind: Cognitive science and human experience. Cambridge, MA: The MIT Press. 